\title{
Application of the Adomian Decomposition Method (ADM) to Solving the Systems of Partial Differential Equations
}

\author{
Justin Mouyedo Loufouilou, Joseph Bonazebi Yindoula, Gabriel Bissanga \\ Department of Exact Sciences, University Marien N'Gouabi, Brazzaville, Congo
}

Email address:

bonayindoula@yahoo.fr (J. B. Yindoula)

\section{To cite this article:}

Justin Mouyedo Loufouilou, Joseph Bonazebi Yindoula, Gabriel Bissanga. Application of the Adomian Decomposition Method (ADM) to Solving the Systems of Partial Differential Equations. International Journal of Applied Mathematics and Theoretical Physics. Vol. 7, No. 1, 2021, pp. 28-39. doi: 10.11648/j.ijamtp.20210701.14

Received: January 15, 2021; Accepted: March 8, 2021; Published: March 30, 2021

\begin{abstract}
Solving systems of partial differential equations (linear or nonlinear) with dirchelet boundary conditions has rarely made use of the Adomian decompositional method. The aim of this paper is to obtain the exact solution of some systems of linear and nonlinear partial differential equations using the adomian decomposition method.After having generated the basic principles of the general theory of this method, five systems of equations are solved, after calculation of the algorithm.Our results suggest that the use of the adomian method to solve systems of partial differential equations is efficient.However, further research should study other systems of linear or nonlinear partial differential equations to better understand the problem of uniqueness of solutions and boundary conditions.
\end{abstract}

Keywords: Adomian Decomposition Method, Systems of Differential Partial Equations, Coupled Partial Differential Equations

\section{Introduction}

Over the past 25 years, the adomian decomposition method (ADM) [1], first introduced by American physicist George Adomian, has been used to efficiently and easily solve a large class of ordinary linear and nonlinear and partial differential equations. In his famous book, Adomian [2, 3] showed the possibility of obtaining explicit solutions to a variety of physical problems. He indicated that no similarity reduction is used to solve Burger's equation, where the explicit solution was obtained using the t-partial solution. In this sense Adomian et al. [4] analyzed mathematical models of the dynamic interaction of the immune response with a population of bacteria, viruses, antigens, or tumor cells. Other researchers, for example by Cherruault et al. [5], Kaya and El-Sayed [6], Biazar et al. [7], Hashim et al. [8], and Lesnic [9].

Subsequently studied analytically and numerically other scientific models. Recently, Sweilam and Khader [10] applied ADM to analyze the nonlinear vibrations of multi-walled carbon nanotubes. In most cases, ADM provides a rapidly converging sequence of approximations, often requiring no more than a few terms for high accuracy. Moreover, the convergence of ADM has been discussed by Cherruault [11], Cherruault et al. [12], Cherruault and Adomian [13], and Cherruault et al. [14]. Moreover, many authors who are interested in this method to solve limit value problems [15] have shown that the ADM method can be used directly without restrictive assumptions, linearization or green functions. For example, Adomian and Rach [16] have shown the efficiency of this method in solving nonlinear BVPs in several dimensions particularly various ordinary and partial differential equations with Dirichlet conditions and Neumanntype boundary conditions. Thus, Adomian by solving the Thomas - Fermi equation subject to the boundary of Dirichlet's conditions to show that his solution depended on the evaluation of the unknown constants of integration by applying the boundary conditions of each determined approximate solution. Many other problems from physics and engineering have been solved by ADM such as the Shawagfeh nonlinear oscillator equation [17], the heat equation of Hadizadeh and Maleknejad [18 ], and Wazwaz's Bratu-type equations [20]. Benabidallah and Cherruault [21-23] also used ADM to solve classes of BVP with Dirichlet boundary conditions subsequently higher 
order nonlinear boundary value problems were investigated by Al-Hayani [ 26], Wazwaz [27, 28] and Hashim [29]. Dehghan [30] applied the ADM to solve a two-dimensional parabolic equation subject to non-standard limit specifications, however little attention has been devoted to the application of $\mathrm{ADM}$ in solving systems of partial differential equations with Neumann boundary conditions, the mathematical difficulties encountered in solving these systems of partial equations this brought led the researchers to develop several techniques to obtain approximate or exact solutions capable of best describing the physical laws and the observed phenomena. This is the case for systems of equations resulting from the Brusselator diffusion-reaction model, the resolution of which appealed among others to the Sumudu method [19] and variational iterations [24, 31, 32]. However, taking into account the difficulties presented in the determination of the exact solution of this system, the decompositional method of Adomian seems to circumvent this one by the use of recursive relations developed in due course, the aim of this paper to determine the exact solutions of some systems of partial differential equations (linear and nonlinear) using the Adomian decompositional method.

\section{The Adomian Decomposition Method}

\subsection{About the Adomian Decomposition Method}

Assume function $\mathrm{u}$ the solution in a real Hilbert space $\mathrm{H}$ of following equation:

$$
A u=f
$$

where $A: H \rightarrow H$ is a linear or a nonlinear operator, $f \in H$ and $u$ is the unknown function. The principle of the ADM is based on the decomposition of the operator $A$ in the following form:

$$
A=L+R+N
$$

The operator A is the linear sum $L+R, N$ nonlinear, $L$ invertible with $L^{-1}$ as inverse. Using that decomposition, equation (1) is equivalent to

$$
u=\theta+L^{-1} f-L^{-1} R u-L^{-1} N u
$$

where $\theta$ verifies $L \theta=0$. (3) is called the Adomian's fundamental equation or Adomian's canonical form. We look for the solution of (1) in a series expansion form $u=$ $\sum_{n=0}^{+\infty} u_{n}$ and we consider $N u=\sum_{n=0}^{+\infty} A_{n}$ where $A_{n}$ are special polynomials of variables $u_{0}, u_{1}, \ldots, u_{n}$ called Adomian polynomials and defined by:

$$
A_{n}=\frac{1}{n !} \frac{d^{n}}{d \lambda^{n}}\left[N\left(\sum_{i=0}^{+\infty} \lambda^{i} u_{i}\right)\right]_{\lambda=0} n=0,1,2, \ldots
$$

where $\lambda$ is a parameter used by "convenience". Thus (3) can be rewritted as follllows:

$$
\sum_{n=0}^{+\infty} u_{n}=\theta+L^{-1} f-L^{-1} R\left(\sum_{n=0}^{+\infty} u_{n}\right)-L^{-1}\left(\sum_{n=0}^{+\infty} A_{n}\right)
$$

If we assume that the series $\sum_{n=0}^{+\infty} u_{n}$ and $\sum_{n=0}^{+\infty} A_{n}$ are convergent, by identification we get the Adomian algorithm:

$$
\left\{\begin{array}{l}
u_{0}=\theta+L^{-1} f=g \\
u_{1}=-L^{-1}\left(R u_{0}\right)-L^{-1} A_{0} \\
\cdot \\
\cdot \\
\cdot \\
u_{n+1}=-L^{-1}\left(R u_{n}\right)-L^{-1} A_{n} \quad n \geqslant 0
\end{array}\right.
$$

In practice it is often difficult to calculate all the terms of an Adomian series, so we approach the series solution by the truncated series: $u=\sum_{i=0}^{n} u_{i}$, where the choice of $\mathrm{n}$ depends on error requirements. If this series converges, the solution of (1) is:

$$
u=\lim _{n \rightarrow+\infty} \sum_{i=0}^{n} u_{i}
$$

\subsection{Remark}

In order overcome the short coming, we assume that $g$ can be divided into the sum of two parts namely $g_{0}$ and $g_{1}$, therefore we get

$$
g=g_{0}+g_{1}
$$

Using the iteration procedure $\mathrm{Eq}$ (6) we suggest the following modification

$$
\left\{\begin{array}{l}
u_{0}=g_{0} \\
u_{1}=g_{1}-L^{-1}\left(R u_{0}\right)-L^{-1} A_{0} \\
u_{n+1}(x, t)=-L^{-1}\left(R u_{n}\right)-L^{-1} A_{n}, n \geq 1
\end{array}\right.
$$

We see that the solution through the modified Adomian decomposition method highly depends upon the choice of $g_{0}$ and $g_{1}$.

\section{Applications}

\subsection{Example 1}

Let us consider the following linear system [10]:

$$
\left\{\begin{array}{l}
\frac{\partial u(x, t)}{\partial t}-\frac{\partial v(x, t)}{\partial x}-u(x, t)+v(x, t)=-2 \\
\frac{\partial v(x, t)}{\partial t}-\frac{\partial u(x, t)}{\partial x}-u(x, t)+v(x, t)=-2 \\
u(x, 0)=1+e^{x} \\
v(x, 0)=-1+e^{x}
\end{array}\right.
$$

where $u(x, t) \subset C^{1}(\Omega)$ et $v(x, t) \subset C^{1}(\Omega), \Omega=[0, L] \times$ $[0, T]$.

$$
\text { Let's take } L_{t}(.)=\frac{\partial(.)}{\partial t}, L_{t}^{-1}(.)=\int_{0}^{t}(.) d s
$$


From (10), we have:

$$
\left\{\begin{array}{l}
u(x, t)=1+e^{x}-2 t+\int_{0}^{t} \frac{\partial v(x, s)}{\partial x} d s+\int_{0}^{t} u(x, s) d s-\int_{0}^{t} v(x, s) d s \\
v(x, t)=-1+e^{x}-2 t+\int_{0}^{t} \frac{\partial u(x, s)}{\partial x} d s+\int_{0}^{t} u(x, s) d s-\int_{0}^{t} v(x, s) d s
\end{array}\right.
$$

Suppose now that the solution $(u(x, t) ; v(x, t))$ of the problem $(10)$ is expressed as form:

$$
\left\{\begin{array}{l}
u(x, t)=\sum_{n=0}^{+\infty} u_{n}(x, t) \\
v(x, t)=\sum_{n=0}^{+\infty} v_{n}(x, t)
\end{array}\right.
$$

Putting (12) into (11) gives

$$
\left\{\begin{array}{l}
\sum_{n=0}^{+\infty} u_{n}(x, t)=1+e^{x}-2 t+\sum_{n=0}^{+\infty} \int_{0}^{t} \frac{\partial\left(v_{n}(x, s)\right)}{\partial x} d s+\sum_{n=0}^{+\infty} \int_{0}^{t}\left(u_{n}(x, s)\right) d s-\sum_{n=0}^{+\infty} \int_{0}^{t}\left(v_{n}(x, s)\right) d s \\
\sum_{n=0}^{+\infty} v_{n}(x, t)=-1+e^{x}-2 t+\sum_{n=0}^{+\infty} \int_{0}^{t} \frac{\partial\left(u_{n}(x, s)\right)}{\partial x} d s+\sum_{n=0}^{+\infty} \int_{0}^{t}\left(u_{n}(x, s)\right) d s-\sum_{n=0}^{+\infty} \int_{0}^{t}\left(v_{n}(x, s)\right) d s
\end{array}\right.
$$

From (13), we get the following Adomian algorithm:

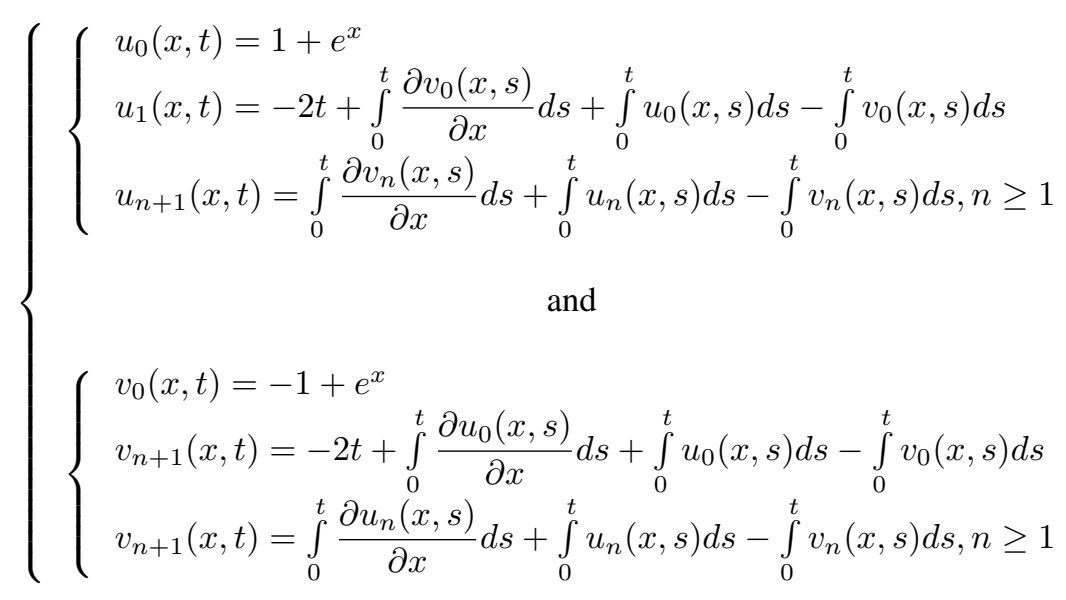

Thus, we obtain:

$$
\left\{\begin{array}{c}
\left\{\begin{array}{c}
u_{1}(x, t)=t e^{x} \\
u_{2}(x, t)=\frac{1}{2} t^{2} e^{x} \\
\vdots \\
u_{n}(x, t)=\frac{1}{n !} t^{n} e^{x}
\end{array}\right. \\
\text { and } \\
\left\{\begin{array}{c}
v_{1}(x, t)=t e^{x} \\
v_{2}(x, t)=\frac{1}{2} t^{2} e^{x} \\
\vdots \\
v_{n}(x, t)=\frac{1}{n !} t^{n} e^{x}
\end{array}\right.
\end{array}\right.
$$


Combining the results obtained above we obtain

$$
\left\{\begin{array}{l}
u(x, t)=1+e^{x}\left(1+t+\frac{1}{2 !} t^{2}+\cdots+\frac{1}{n !} t^{n}+\cdots\right) \\
v(x, t)=-1+e^{x}\left(1+t+\frac{1}{2 !} t^{2}+\cdots+\frac{1}{n !} t^{n}+\cdots\right)
\end{array}\right.
$$

The solution of the problem (10) is

$$
\left\{\begin{array}{l}
u(x, t)=1+e^{x+t} \\
v(x, t)=-1+e^{x+t}
\end{array}\right.
$$

\subsection{Example 2}

Let us now consider the following system of linear partial differential equations:

$$
\left\{\begin{array}{l}
\frac{\partial u(x, t)}{\partial t}+\frac{\partial v(x, t)}{\partial x}-u(x, t)-v(x, t)=0 \\
\frac{\partial v(x, t)}{\partial t}+\frac{\partial u(x, t)}{\partial x}-u(x, t)-v(x, t)=0 \\
u(x, 0)=\sinh x \\
v(x, 0)=\cosh x
\end{array}\right.
$$

where $u(x, t) \subset C^{1}(\Omega)$ and $v(x, t) \subset C^{1}(\Omega), \Omega=[0, T] \times[0, T]$.

Operating with $L_{t}^{-1}()=.\int_{0}^{t}() d$.$s on (18)$, we obtain

$$
\left\{\begin{array}{l}
u(x, t)=\sinh x-\int_{0}^{t} \frac{\partial v(x, s)}{\partial x} d s+\int_{0}^{t}(u(x, s)+v(x, s)) d s \\
v(x, t)=\cosh x-\int_{0}^{t} \frac{\partial u(x, s)}{\partial x} d s+\int_{0}^{t}(u(x, s)+v(x, s)) d s
\end{array}\right.
$$

Let's now we suppose that the solution $(u(x, t) ; v(x, t))$ of the problem $(18)$ has the following form:

$$
\left\{\begin{array}{l}
u(x, t)=\sum_{n=0}^{+\infty} u_{n}(x, t) \\
v(x, t)=\sum_{n=0}^{+\infty} v_{n}(x, t)
\end{array}\right.
$$

from (19) using (20), we have

$$
\left\{\begin{array}{c}
\sum_{n=0}^{+\infty} u_{n}(x, t)=\sinh x-\int_{0}^{t} \frac{\partial\left(\sum_{n=0}^{+\infty} v_{n}(x, s)\right)}{\partial x} d s+\int_{0}^{t}\left(\sum_{n=0}^{+\infty} u_{n}(x, s)+\sum_{n=0}^{+\infty} v_{n}(x, s)\right) d s \\
\sum_{n=0}^{+\infty} v_{n}(x, t)=\cosh x-\int_{0}^{t} \frac{\partial\left(\sum_{n=0}^{+\infty} u_{n}(x, s)\right)}{\partial x} d s+\int_{0}^{t}\left(\sum_{n=0}^{+\infty} u(x, s)+\sum_{n=0}^{+\infty} v_{n}(x, s)\right) d s
\end{array}\right.
$$


Therefore, the pair of zeroth components is given by

$$
\left\{\begin{array}{l}
\left\{\begin{array}{l}
u_{0}(x, t)=\sinh x \\
u_{n}(x, t)=-\int_{0}^{t} \frac{\partial\left(v_{n}(x, s)\right)}{\partial x} d s+\int_{0}^{t}\left(u_{n}(x, s)+v_{n}(x, s)\right) d s
\end{array}\right. \\
\text { and } \\
\left\{\begin{array}{l}
v_{0}(x, t)=\cosh x \\
v_{n}(x, t)=\cosh x-\int_{0}^{t} \frac{\partial\left(u_{n}(x, s)\right)}{\partial x} d s+\int_{0}^{t}\left(u_{n}(x, s)+v_{n}(x, s)\right) d s
\end{array}\right.
\end{array}\right.
$$

Consequently, we obtain

$$
\left\{\begin{array}{l}
\left\{\begin{array}{l}
u(x, t)=\sinh x \\
u_{1}(x, t)=t \cosh x \\
u_{2}(x, t)=\frac{t^{2}}{2 !} \sinh x \\
u_{3}(x, t)=\frac{t^{3}}{3 !} \cosh x \\
\vdots \\
\text { and } \\
v_{0}(x, t)=\cosh x \\
v_{1}(x, t)=t \sinh x \\
v_{2}(x, t)=\frac{t^{2}}{2 !} \cosh x \\
v_{3}(x, t)=\frac{t^{3}}{3 !} \sinh x \\
\vdots
\end{array}\right.
\end{array}\right.
$$

Rearranging the results obtained previously gives

$$
\left\{\begin{array}{l}
u(x, t)=\left(1+\frac{t^{2}}{2 !}+\cdots\right) \sinh x+\left(t+\frac{t^{3}}{3 !}+\cdots\right) \cosh x \\
v(x, t)=\left(1+\frac{t^{2}}{2 !}+\cdots\right) \cosh x+\left(t+\frac{t^{3}}{3 !}+\cdots\right) \sinh x
\end{array}\right.
$$

We obtain the solution of the problem (18) :

$$
\left\{\begin{array}{l}
u(x, t)=\sinh (x+t) \\
v(x, t)=\cosh (x+t)
\end{array}\right.
$$

\subsection{Example 3}

Let us now consider the following system of non linear partial differential equations :

$$
\left\{\begin{array}{l}
\frac{\partial u(x, t)}{\partial t}+2 \frac{\partial u(x, t)}{\partial x} v(x, t)-u(x, t)=2 \\
\frac{\partial v(x, t)}{\partial t}-3 \frac{\partial v x, t)}{\partial x} u(x, t)-v(x, t)=3 \\
u(x, 0)=e^{x} \\
v(x, 0)=e^{-x}
\end{array}\right.
$$


where $u(x, t) \subset C^{1}(\Omega)$ and $v(x, t) \subset C^{1}(\Omega), \Omega=[0, T] \times[0, T]$.

Operating with $L_{t}^{-1}()=.\int_{0}^{t}() d$.$s on (26)$, we get

$$
\left\{\begin{array}{c}
u(x, t)=e^{x}+2 t-2 \int_{0}^{t} N(x, s) d s+\int_{0}^{t} u(x, s) d s \\
v(x, t)=e^{-x}+3 t+3 \int_{0}^{t} M(x, s) d s-\int_{0}^{t} v(x, s) d s
\end{array}\right.
$$

Where $N(u, v)=\frac{\partial u(x, t)}{\partial x} \times v(x, t)$ and $M(u, v)=\frac{\partial v(x, t)}{\partial x} \times u(x, t)$.

According to the ADM, we assume that the solution of (26) is expressed as

$$
\left\{\begin{array}{l}
u(x, t)=\sum_{n=0}^{+\infty} u_{n}(x, t) \\
v(x, t)=\sum_{n=0}^{+\infty} v_{n}(x, t)
\end{array}\right.
$$

and

$$
\left\{\begin{array}{l}
A_{n}(x, t)=N(u, v)=\frac{1}{n !} \frac{d^{n}}{d \lambda^{n}}\left[\left(\sum_{n=0}^{+\infty} \lambda^{i} u_{i}\right)_{x}^{\prime}\left(\sum_{n=0}^{+\infty} \lambda^{i} v_{i}\right)\right]_{\lambda=0} ; n=0,1, \ldots \\
B_{n}(x, t)=M(u, v)=\frac{1}{n !} \frac{d^{n}}{d \lambda^{n}}\left[\left(\sum_{n=0}^{+\infty} \lambda^{i} v_{i}\right)_{x}^{\prime}\left(\sum_{n=0}^{+\infty} \lambda^{i} u_{i}\right)\right]_{\lambda=0}
\end{array}\right.
$$

Using the (ADM), the equation (27) can be written as follows:

$$
\left\{\begin{array}{c}
\sum_{n=0}^{+\infty} u_{n}(x, t)=e^{x}+2 t-2 \int_{0}^{t}\left(\sum_{n=0}^{+\infty} A_{n}(x, s)\right) d s+\int_{0}^{t}\left(\sum_{n=0}^{+\infty} u_{n}(x, s)\right) d s \\
\sum_{n=0}^{+\infty} v_{n}(x, t)=e^{-x}+3 t+3 \int_{0}^{t}\left(\sum_{n=0}^{+\infty} B_{n}(x, s)\right) d s-\int_{0}^{t}\left(\sum_{n=0}^{+\infty} v_{n}(x, s)\right) d s
\end{array}\right.
$$

So, the Adomian algorithm is:

$$
\left\{\begin{array}{l}
\left\{\begin{array}{l}
u_{0}(x, t)=e^{x} \\
u_{1}(x, t)=2 t-2 \int_{0}^{t} A_{0}(x, s) d s+\int_{0}^{t} u_{0}(x, s) d s \\
u_{n+1}(x, t)=-2 \int_{0}^{t} A_{n}(x, s) d s+\int_{0}^{t} u_{n}(x, s) d s, n \geq 1
\end{array}\right. \\
\left\{\begin{array}{l}
v_{0}(x, t)=e^{-x} \text { and } \\
v_{1}(x, t)=3 t+3 \int_{0}^{t} B_{0}(x, s) d s-\int_{0}^{t} v_{0}(x, s) d s \\
v_{n+1}(x, t)=3 \int_{0}^{t} B_{n}(x, s) d s-\int_{0}^{t} v_{n}(x, s) d s, n \geq 1
\end{array}\right.
\end{array}\right.
$$


(29)and (31) result to:

$$
\left\{\begin{array}{l}
\left\{\begin{array}{l}
A_{0}=N\left(u_{0}, v_{0}\right)=\frac{\partial u_{0}(x, t)}{\partial x} \times v_{0}(x, t)=1, \\
u_{1}(x, t)=t e^{x} \\
A_{n}=0, n \geq 1 \\
u_{n}(x, t)=\frac{t^{n}}{n !} e^{x}, n \geq 2
\end{array}\right. \\
\left\{\begin{array}{l}
B_{0}=M\left(u_{0}, v_{0}\right)=\frac{\partial v_{0}(x, t)}{\partial x} \times u_{0}(x, t)=-1 \\
v_{1}(x, t)=-t e^{-x} \\
B_{n}=0, n \geq 1 \\
v_{n}(x, t)=\frac{(-t)^{n}}{n !} e^{-x}, n \geq 2 .
\end{array}\right.
\end{array}\right.
$$

We obtain the solution of the problem (26)

$$
\left\{\begin{array}{l}
u(x, t)=\lim _{n \rightarrow+\infty} \sum_{i=0}^{n} \frac{t^{n}}{n !} e^{x}=e^{x+t} \\
v(x, t)=\lim _{n \rightarrow+\infty} \sum_{i=0}^{n} \frac{(-t)^{n}}{n !} e^{-x}=e^{-t-x}
\end{array}\right.
$$

\subsection{Example 4}

Let us consider an other system of non linear partial differential equations:

$$
\left\{\begin{array}{l}
\frac{\partial u(x, t)}{\partial t}-\frac{\partial^{2} u(x, t)}{\partial x^{2}}-2 u(x, t) \frac{\partial u(x, t)}{\partial x}+v(x, t) u(x, t)=0 \\
\frac{\partial v(x, t)}{\partial t}-\frac{\partial^{2} v(x, t)}{\partial x^{2}}-2 v(x, t) \frac{\partial v(x, t)}{\partial x}+v(x, t) u(x, t)=0 \\
u(x, 0)=\sin x \\
v(x, 0)=\sin x
\end{array}\right.
$$

Operating $L_{t}^{-1}()=.\int_{0}^{t}() d$.$s on (34)$, leads to:

$$
\left\{\begin{array}{l}
u(x, t)=\sin x+\int_{0}^{t} \frac{\partial^{2} u(x, s)}{\partial x^{2}} d s+2 \int_{0}^{t} u(x, s) \times \frac{\partial u(x, s)}{\partial x} d s-\int_{0}^{t} \frac{\partial}{\partial x}[v(x, s) u(x, s)] d s \\
v(x, t)=\sin x+\int_{0}^{t} \frac{\partial^{2} v(x, s)}{\partial x^{2}} d s+2 \int_{0}^{t} v(x, s) \times \frac{\partial v(x, s)}{\partial x} d s-\int_{0}^{t} \frac{\partial}{\partial x}[v(x, s) u(x, s)] d s
\end{array}\right.
$$

$\Longleftrightarrow$

$$
\left\{\begin{array}{l}
u(x, t)=\sin x+\int_{0}^{t} \frac{\partial^{2} u(x, s)}{\partial x^{2}} d s+2 \int_{0}^{t} M(u, v) d s-\int_{0}^{t} P(u, v) d s \\
v(x, t)=\sin x+\int_{0}^{t} \frac{\partial^{2} v(x, s)}{\partial x^{2}} d s+2 \int_{0}^{t} N(u, v) d s-\int_{0}^{t} P(u, v) d s
\end{array}\right.
$$

Where

$$
\left\{\begin{array}{l}
M(u, v)=u(x, t) \times \frac{\partial u(x, t)}{\partial x} \\
N(u, v)=v(x, t) \times \frac{\partial v(x, t)}{\partial x} \\
P(u, v)=\frac{\partial}{\partial x}[v(x, t) u(x, t)]
\end{array}\right.
$$


According to the ADM, we suppose that the solution of (34) has the following form

$$
(u(x, t) ; v(x, t))=\left(\sum_{n=0}^{+\infty} u_{n}(x, t) ; \sum_{n=0}^{+\infty} v_{n}(x, t)\right)
$$

and

$$
\left\{\begin{array}{c}
M(u, v)=A_{n}(x, t)=\frac{1}{n !} \frac{d^{n}}{d \lambda^{n}}\left[\left(\sum_{n=0}^{+\infty} \lambda^{i} u_{i}\right)\left(\sum_{n=0}^{+\infty} \lambda^{i} u_{i}\right)_{x}^{\prime}\right]_{\lambda=0} ; n=0,1,2, \ldots \\
M(u, v)=B_{n}(x, t)=\frac{1}{n !} \frac{d^{n}}{d \lambda^{n}}\left[\left(\sum_{n=0}^{+\infty} \lambda^{i} v_{i}\right)\left(\sum_{n=0}^{+\infty} \lambda^{i} v_{i}\right)_{x}^{\prime}\right]_{\lambda=0} ; n=0,1,2, \ldots . . \\
P(u, v)=C_{n}(x, t)=\frac{1}{n !} \frac{d^{n}}{d \lambda^{n}}\left[\left(\sum_{n=0}^{+\infty} \lambda^{i} u_{i} \sum_{n=0}^{+\infty} \lambda^{i} v_{i}\right)_{x}^{\prime}\right]_{\lambda=0} ; n=0,1,2, \ldots
\end{array}\right.
$$

We obtain

$$
\left\{\begin{array}{l}
\sum_{n=0}^{+\infty} u_{n}(x, t)=\sin x+\int_{0}^{t} \frac{\partial^{2}\left(\sum_{n=0}^{+\infty} u_{n}(x, s)\right)}{\partial x^{2}} d s+2 \int_{0}^{t} A_{n}(x, s) d s-\int_{0}^{t} C_{n}(x, s) d s \\
\sum_{n=0}^{+\infty} v_{n}(x, t)=\sin x+\int_{0}^{t} \frac{\partial^{2}\left(\sum_{n=0}^{+\infty} v_{n}(x, s)\right)}{\partial x^{2}} d s+2 \int_{0}^{t} B_{n}(x, s) d s-\int_{0}^{t} C_{n}(x, s) d s
\end{array}\right.
$$

and the Adomian algorithm can be written as:

$$
\left\{\begin{aligned}
u_{0}(x, t)= & \sin x \\
u_{n+1}(x, t)= & \int_{0}^{t} \frac{\partial^{2} u_{n}(x, s)}{\partial x^{2}} d s+2 \int_{0}^{t} A_{n}(x, s) d s-\int_{0}^{t} C_{n}(x, s) d s, n \geq 0 \\
v_{0}(x, t)= & \sin x \\
v_{n+1}(x, t) & \int_{0}^{t} \frac{\partial^{2} v_{n}(x, s)}{\partial x^{2}} d s+2 \int_{0}^{t} B_{n}(x, s) d s-\int_{0}^{t} C_{n}(x, s) d s, n \geq 0
\end{aligned}\right.
$$

(39) and (41) result to:

$$
\left\{\begin{array}{l}
A_{0}(x, t)=u_{0}(x, t) \times \frac{\partial u_{0}(x, t)}{\partial x}=(\sin x) \frac{\partial}{\partial x}(\sin x)=\cos x \sin x \\
B_{0}(x, t)=v_{0}(x, t) \times \frac{\partial v_{0}(x, t)}{\partial x}=(\sin x) \times \frac{\partial}{\partial x}(\sin x)=\cos x \sin x \\
C_{0}(x, t)=\frac{\partial}{\partial x}\left(v_{0}(x, t) u_{0}(x, t)\right)=\frac{\partial}{\partial x}\left(\sin ^{2} x\right)=2 \cos x \sin x \\
u_{1}(x, t)=-t \sin x \\
v_{1}(x, t)=-t \sin x
\end{array}\right.
$$

If $n=1$, we obtain:

$$
\left\{\begin{array}{l}
A_{1}(x, t)=u_{0} u_{1 x}+u_{1} u_{0 x} \\
B_{1}(x, t)=v_{0} v_{1 x}+v_{1} v_{0 x} \\
C_{1}(x, t)=u_{1 x} v_{0}+u_{0 x} v_{1}+u_{1} v_{0 x}+u_{0} v_{1 x} \\
u_{2}=\frac{t^{2}}{2 !} \sin x \\
v_{2}=\frac{t^{2}}{2 !} \sin x
\end{array}\right.
$$

Similarly, the pairs can be expressed as:

$$
\left\{\begin{array}{l}
u_{3}(x, t)=-\frac{t^{3}}{3 !} \sin x \\
v_{3}(x, t)=-\frac{t^{3}}{3 !} \sin x
\end{array}\right.
$$


and

$$
\left\{\begin{array}{l}
u_{4}(x, t)=\frac{t^{4}}{4 !} \sin x \\
v_{4}(x, t)=\frac{t^{4}}{4 !} \sin x
\end{array}\right.
$$

$$
\left\{\begin{array}{l}
u_{n}(x, t)=\frac{1}{n !}(-t)^{n} \sin x \\
v_{n}(x, t)=\frac{1}{n !}(-t)^{n} \sin x
\end{array} \forall n \in N .\right.
$$

Combining the results obtained above, we obtain:

$$
\left\{\begin{array}{l}
u(x, t)=\left(1+(-t)+\frac{(-t)^{2}}{2 !}+\frac{(-t)^{3}}{3 !}+\ldots+\frac{1}{n !}(-t)^{n}+. .\right) \sin x \\
v(x, t)=\left(1+(-t)+\frac{(-t)^{2}}{2 !}+\frac{(-t)^{3}}{3 !}+\ldots+\frac{1}{n !}(-t)^{n}+. .\right) \sin x
\end{array}\right.
$$

The solution of the problem $(34)$ is

$$
\left\{\begin{array}{l}
u(x, t)=e^{-t} \sin x \\
v(x, t)=e^{-t} \sin x
\end{array}\right.
$$

\subsection{Example 5}

Finally, consider the system nonlinear coupled partial differential equations [10]

$$
\left\{\begin{array}{l}
\frac{\partial u(x, y, t)}{\partial t}-\frac{\partial v(x, y, t)}{\partial x} \frac{\partial w(x, y, t)}{\partial y}=1 \\
\frac{\partial v(x, y, t)}{\partial t}-\frac{\partial w(x, y, t)}{\partial x} \frac{\partial u(x, y, t)}{\partial y}=5 \\
\frac{\partial w(x, y, t)}{\partial t}-\frac{\partial u(x, y, t)}{\partial x} \frac{\partial v(x, y, t)}{\partial y}=5
\end{array}\right.
$$

Where the initial conditions are

$$
\left\{\begin{array}{l}
u(x, y, t)=x+2 y \\
v(x, y, t)=x-2 y \\
w(x, y, t)=-x+2 y
\end{array}\right.
$$

with

$$
\left\{\begin{array}{l}
N_{1}(u, w)=\frac{\partial v(x, y, t)}{\partial x} \frac{\partial w(x, y, t)}{\partial y} \\
N_{2}(u, w)=\frac{\partial w(x, y, t)}{\partial x} \frac{\partial u(x, y, t)}{\partial y} \\
N_{3}(u, v)=\frac{\partial u(x, y, t)}{\partial x} \frac{\partial v(x, y, t)}{\partial y}
\end{array}\right.
$$


Integrating the system with respect to $t$, gives:

$$
\left\{\begin{array}{l}
u(x, y, t)=x+2 y+t+\int_{0}^{t} N_{1}(u, w) d s \\
v(x, y, t)=x-2 y+5 t+\int_{0}^{t} N_{2}(u, w) d s \\
w(x, y, t)=-x+2 y+5 t+\int_{0}^{t} N_{3}(u, w) d s
\end{array}\right.
$$

Let's pose

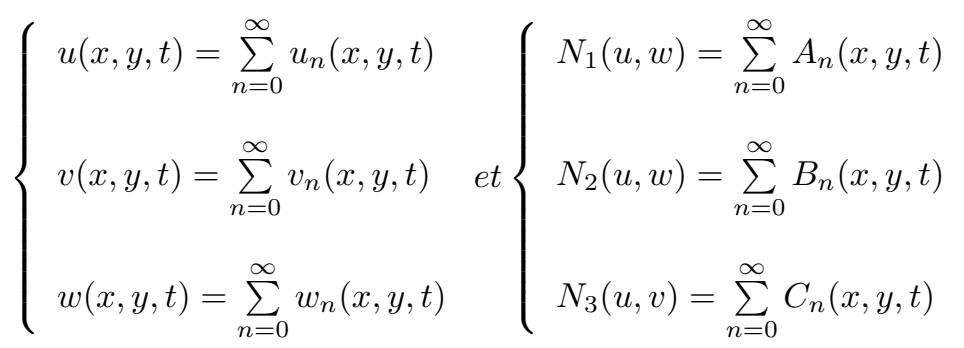

We have the following Adomian algorithm:

$$
\left\{\begin{array}{l}
\left\{\begin{array}{l}
u_{0}(x, y, t)=x+2 y+3 t \\
u_{1}(x, y, t)=-2 t+\int_{0}^{t} A_{0}(x, y, t) d s \\
u_{n}(x, y, t)=\int_{0}^{t} A_{n-1}(x, y, s) d s, \forall n \geq 2
\end{array}\right. \\
\left\{\begin{array}{l}
v_{0}(x, y, t)=x-2 y+3 t \\
v_{1}(x, y, t)=2 t+\int_{0}^{t} B_{0}(x, y, s) d s \\
v_{n}(x, y, t)=\int_{0}^{t} B_{n-1}(x, y, t) d s, \forall n \geq 2 \\
w_{0}(x, y, t)=-x+2 y+3 t \\
w_{1}(x, y, t)=2 t+\int_{0}^{t} C_{0}(x, y, t) d s \\
w_{n}(x, y, t)=\int_{0}^{t} C_{n-1}(x, y, t) d s, \forall n \geq 2
\end{array}\right.
\end{array}\right.
$$

Which gives us

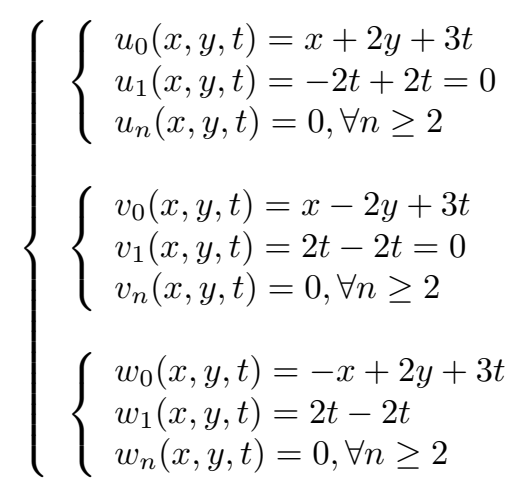


Therefore, the solutions of this system of non linear coupled partial differential equations are

$$
\left\{\begin{array}{l}
u(x, y, t)=u_{0}(x, y, t)=x+2 y+3 t \\
v(x, y, t)=v_{0}(x, y, t)=x-2 y+3 t \\
w(x, y, t)=w_{0}(x, y, t)=-x+2 y+3 t
\end{array}\right.
$$

\section{Conclusion}

The findings of this article which focused on the exact solution of two systems of linear partial differential equations, two systems of nonlinear partial differential equations and a system of coupled nonlinear partial differential equations, show that calculus of Adomian algorithm is fast and it results in exact analytical solutions.

Determining the exact solutions for all these systems proves the efficiency of the method. However,further research should investigate other systems of linear and non linear partial differential equations in order to better identify the problems posed by the implementation of this method.

\section{References}

[1] K. Abbaoui, Les fondements de la méthode décompositionnelle d'Adomian et application à la résolution de problèmes issus de la biologie et de la médécine. Thèse de doctorat de l'Université Paris VI. Octobre 1995.

[2] K. ABBAOUI and Y. CHERRUAULT, "Convergence of Adomian method applied to differentiel equations." Math Comput Modelling (28.3). pp 103-109, 1994.

[3] K. ABBAOUI and Y. CHERRUAULT," The Decomposition Adomian method applied to the nonliear equations.” Math Comput Modelling (20.9). pp 60-73 1994.

[4] K. ABBAOUI and Y. CHERRUAULT, " The Decomposition Adomian method applied to the Cauchy problem.” Kybernetes (28.1) pp 68-74, 1999.

[5] Y. Cherruault, M. Inc, K. Abbaoui, On the solution of the nonlinear Korteweg-de Vries equation by the decomposition method, Kybernetes 31 (2002) 766-772.

[6] D. Kaya, S.M. El-Sayed, A numerical method for solving Jaulent-Miodek equation, Phys. Lett. A 318 (2003) 345353.

[7] J. Biazar, M. Ilie, A. Khoshkenar, A new approach to the solution of the prey and predator problem and comparison of the results with the Adomian method, Appl. Math. Comput. 171 (2005) 486-491.

[8] I. Hashim, M.S.M. Noorani, R. Ahmad, S.A. Bakar, E.S. Ismail, A.M. Zakaria, Accuracy of the Adomian decomposition method applied to the Lorenz system, Chaos Solitons Fractals 28 (2006) 1149-1158.

[9] D. Lesnic, Blow-up solutions obtained using the decomposition method, Chaos Solitons Fractals 28 (2006) 776-787.

[10] N.H. Sweilam, M.M. Khader, Approximate solutions to the nonlinear vibrations of multiwalled carbon nanotubes using Adomian decomposition method, Appl. Math. Comput. 217 (2010) 495-505.

[11] Y. Cherruault, Convergence of Adomian's method, Kybernetes 18 (1989) 31-38.

[12] Y. Cherruault, G. Adomian, Decomposition method, a new proof of convergence, Math. Comput. Modelling 18 (1993) 103-106.

[13] N. NGARASTA, B.SOME, K. ABBAOUI and Y. CHERRUAULT, "New numerical study of Adomian method applied to a diffusion model." Kybernetes. Vol. 31, n 1 pp 61-75, 2002.

[14] Y. Cherruault, G. Adomian, K. Abbaoui, R. Rach, Further remarks on convergence of decomposition method, Int. J. Bio-Med. Comput. 38 (1995) 89-93

[15] G. Adomian, R. Rach, Analytic solution of nonlinear boundary-value problems in several dimensions by decomposition, J. Math. Anal. Appl. 174 (1993) 118137.

[16] Chengri Jin, Mingzhu Liu. "A new modification of Adomian decomposition method to solving a kind of evolution equation". Applied Mathematicals and Computation 169 (2005) 953-962.

[17] Hassan Eltayeb and Adem Kihçman. Application of Sumudu Decomposition Method to Solve Nonlinear System of Partial Differential Equations, Hindawi Publishing Corporation,Vol.2012,Article ID412948,13 pages doi:10.11554/2012/412948

[18] M. Hadizadeh, K. Maleknejad, On the decomposition method to the heat equation with nonlinear and non-local boundary conditions, Kybernetes 27 (1998) 426-434.

[19] A. M. Wazwaz, "Partial Differential Equations and Solitary Waves Theory." Nonlinear Physical science.

[20] Y. Cherruault, G. Saccamondi, B. Some, New results for convergence of Adomian's method applied to integral equations, Math. Comput. Modelling 16 (1992) 85-93. 
[21] A. M. Wazwaz, "Partial Differential Equations and Solitary Waves Theory." Nonlinear Physical science.

[22] G. BISSANGA, A. K. NSEMI. ”Application of Adomian decomposition method to solving the Van der Pol equation and comparison with the regular perturbation method." Proccedinds of Five international workshop on contemporary problems in mathematical physics, Cotonou-Benin, eds. J. Govaerts, M. N. HOUKOUNOU (International Chair in Mathematical Physics and Applications, ICMP-Unesco Chair. University of Abomey-Calavy.072 Bp 50 Cotonou, Republic of Benin, December 2008).

[23] Pierre BAKI-TANGOU, G. BISSANGA. "Application of Adomian decomposition merhod to solving the DuffingVan Der Pol equation. Communications in Mathematical Analysis. Vol. 4, N² pp30-40 (2008).

[24] JH He Méthode d'itération variationnelle pour les systèmes différentiels ordinaires autonomes Appl. Math. Comput. , 114 ( 2-3 ) ( 2000 ), pp. 115 - 123

[25] W. Al-Hayani, Adomian decomposition method with Green's function for sixth-order boundary value problems, Comput. Math. Appl. 61 (2011) 1567-1575
[26] A.M. Wazwaz, The numerical solution of fifth-order boundary value problems by the decomposition method, J. Comput. Appl. Math. 136 (2001) 259-270.

[27] A.M. Wazwaz, A note on using Adomian decomposition method for solving boundary value problems, Found. Phys. Lett. 13 (2000) 493-498.

[28] I. Hashim, Adomian decomposition method for solving BVPs for fourth-order integro-differential equations, J. Comput. Appl. Math. 193 (2006) 658-664.

[29] M. Dehghan, Application of the Adomian decomposition method for two-dimensional parabolic equation subject to nonstandard boundary specifications, Appl. Math. Comput. 157 (2004) 549-560

[30] G. Adomian, R. Rach, Equality of partial solutions in the decomposition method for linear or nonlinear partial differential equations, Comput. Math. Appl. 19 (1990) 9-12.

[31] E. Hetmaniok, D. Slota, R. Witula, A. Zielonka, Comparison of the Adomian decomposition method and the variational iteration method in solving the moving boundary problem, Comput. Math. Appl. 61 (2011) 1931-1934. 